\title{
On the electromagnetic origin of inertia and inertial mass
}

\author{
Alexandre A. Martins: \\ Institute for Plasmas and Nuclear Fusion, \\ Instituto Superior Tecnico, \\ Av. Rovisco Pais, 1049-001 Lisboa, Portugal \\ Mario J. Pinheird ${ }^{\dagger}$ \\ Department of Physics and Institute for Plasmas and Nuclear Fusion, \\ Instituto Superior Tecnico, Av. Rovisco Pais, 8 1049-001 Lisboa, Portugal
}

(Dated: February 5, 2008)

\begin{abstract}
We address the problem of inertial property of matter through analysis of the motion of an extended charged particle. Our approach is based on the continuity equation for momentum (Newton's second law) taking due account of the vector potential and its convective derivative. We obtain a development in terms of retarded potentials allowing an intuitive physical interpretation of its main terms. The inertial property of matter is then discussed in terms of a kind of induction law related to the extended charged particle's own vector potential. Moreover, it is obtained a force term that represents a drag force acting on the charged particle when in motion relatively to its own vector potential field lines. The time rate of variation of the particle's vector potential leads to the acceleration inertia reaction force, equivalent to the Schott term responsible for the source of the radiation field. We also show that the velocity dependent term of the particle's vector potential is connected with the relativistic increase of mass with velocity and generates a longitudinal stress force that is the source of electric field lines deformation. In the framework of classical electrodynamics, we have shown that the electron mass has possibly a complete electromagnetic origin and the obtained covariant equation solves the " $4 / 3$ mass paradox" for a spherical charge distribution.
\end{abstract}

PACS numbers: 03.50.De;03.50.-z;03.30.+p;01.55.+b

Keywords: Classical electromagnetism, Maxwell equations; classical field theories; Special relativity; General physics; Electromagnetic propulsion

\section{INTRODUCTION}

Advanced propulsion systems are necessary in order to open up the cosmos to robotic and future human exploration. This quest motivates a renewed interest in studies about the inertial property of matter suggested qualitatively by Galileu in his writings and later quantified by Newton [1]. However its conceptualization still remains as an unclear resistance of mass to changes of its state of motion [2].

Several approaches were proposed, among them: i) linking inertia with gravitational interactions with the rest of the universe [3, 4, 5]; ii) the evolving notion of a energy replenishing vacuum motivate other line of research sustained in the hypothesis that inertial forces result from an interaction of matter with electromagnetic fluctuations of the zero point field 6, 7, 8]; iii) also, other line of thought attribute inertia to the result of the particle interaction with its own field [9, 10, 14].

Experimental studies of electrically charged particles animated of high velocities have lead physicists to introduce the notion of electromagnetic inertia besides mechanical inertia. J. J. Thomson (that discovered the electron in studying cathode rays [15]) was the first to introduce the idea of a supplementary inertia with constant magnitude for a charge $q$ with radius $R$ in a medium of magnetic permeability $\mu$, to be summed up with the mechanical mass $m$ such that $m+4 \mu q /(15 R)$ [16] (see Ref. [17] for a deep historical account). Inspired probably on Stokes [18] finding that a body moving in water seems to acquire a supplementary mass, Thomson built a hydrodynamical model with tubes of force displacing the ether.

These studies have not yet achieved a clear and concise explanation of the phenomenon, although different approaches to the classical model of the electron in vacuum may contribute to clarify hidden aspects of the problem [19]. Until now there is no experimental support of Mach's principle as a recent experimental test using nuclear-spinpolarized ${ }^{9} \mathrm{Be}^{+}$ions gives null result on spatial anisotropy and thus supporting Local Lorentz Invariance [20]. This

*Electronic address: aam@ist.utl.pt

†Electronic address: mpinheiro@ist.utl.pt 
supports our viewpoint that inertia is a local phenomena and it is along this epistemological basis that we discuss here the inertia property of matter in terms of an interaction of material particle's own vector potential with mechanical momentum (in accelerating or decelerating particles). This is substantially the problem of the self-force (or radiationreaction force), the remarkable phenomena of the interaction of the charged particle on itself. The nonrelativistic form of this force was obtained primarily by Abraham (1903) [10, 21] and Lorentz (1904) [9], while later Dirac obtained the covariant relativistic expression of the self-force [22].

The present paper introduces a new approach to the classical electron problem yielding a pre-relativistic treatment of the motion of an "electron-like" extended charged particle. Previously different types of charged distribution and approaches were considered [23, 24, 25]. The extended object approach considers an extended charged object of finite size $\epsilon$ and Ori and Rosenthal [12, 13] obtained a universal (i.e., shape-independent), consistent interpretation of the self-force in terms of classical electrodynamics, solving the " $4 / 3$ problem" for a spherical charge distribution.

On the basis of the convective derivative terms we attempt to elucidate the physical meaning of the derived terms obtained by using the Lorentz's procedure at the lowest order. In the framework of our classical electrodynamic approach, the " $4 / 3$ problem" can be solved considering the total electromagnetic force acting on an extended-charge particle. This is done by introducing the electromagnetic vector and scalar potentials $(\mathbf{A}, \phi)$ and calculating the moving self-field referring to field points that move along with the particle. Using such a procedure the electron mass and inertia can be shown to have a complete electromagnetic origin, a conclusion obtained a long time ago by Fermi [59] carrying out a variational calculation. Our driving motive can be found in Heaviside's idea that "It seems [...] not unlikely that in discussing purely electromagnetic speculations, one may be within a stone's throw of the explanation of gravitation all the time" [26].

\section{THE ELECTROMAGNETIC ORIGIN OF INERTIA}

Newton's first law - the law of inertia - states that a body remains at rest or in motion with the same speed and in the same direction unless acted upon by a force. Newton's second law of motion tells us that to overcome inertia the applied force needs to have the magnitude of the inertia force. As stated by Newton's third law, for every action (acceleration) there is a reaction (inertia). However, these two forces do not cancel each other since velocity has to change for the effect to take place due to the retarded fields emanating from an accelerated charge.

In the frame of the Lagrangian formalism of a charged point particle the generalized (canonical) momentum must be $\mathbf{p}=m \mathbf{v}+q \mathbf{A}$. Whenever the particle is not subject to an external force, it is $\mathbf{p}$ rather than $m \mathbf{v}$ that is conserved. Maxwell advocated in 1865 that the vector potential could be seen as a stored momentum per unit charge, and Thomson in 1904 interpreted A as a field momentum per unit charge. More recently, Mead [27] derived standard results of electromagnetic theory from the direct interaction of macroscopic quantum systems assuming solely the Einstein-de Broglie relations, the discrete nature of charge, the Green's function for the vector potential, and the continuity of the wave function - without any reference to Maxwell's equations. Holding an opposite view are Heaviside and Hertz who envisaged the vector potential as merely an auxiliary artifact to computation (see Refs. 228, 29, 30, 31, 32, 33, 34, 35]). The physicality of the vector potential is now well proven experimentally [36] and it was shown that in certain welldefined situations are measurable and possess a topology transforming according to the SU(2) group [37].

In this paper we discuss inertia in terms of the "potential momentum", or vector potential created by the particle, as the primary source for the inertia force. Within the frame of quantum mechanics it is now clear that the potential functions $(\mathbf{A}, \phi)$ emerge as more fundamental quantities than the $(\mathbf{E}, \mathbf{B})$ fields, predicting certain quantum interference effects, like the Aharonov-Bohm effect and the single-leg electron interferometer effect known as the Josephson effect.

It is known that any charged particle in motion constitute an electric current with an associated "potential momentum", A. When the velocity is uniform $\mathbf{A}$ is constant in magnitude and no "potential momentum" will be exchanged between the field and the particle. If the velocity varies, however, the difference in "potential momentum" caused by the resulting acceleration will exert a force on the particle itself which will be opposed to the external applied force.

Let us consider one positive particle (with non-negligible dimensions and radius $R$ ) submitted to an external acceleration and in a rectilinear motion. The particle acquires a velocity in the same direction, constituting an electric current $I$ (and related to the vector density of charge $\mathbf{J}=\rho \mathbf{v}$ ), with an associated potential vector $\mathbf{A}$ in the same direction of velocity. The retarded field is given by:

$$
\mathbf{A}(\mathbf{x}, t)=\frac{\mu_{0}}{4 \pi} \iiint_{V} \frac{\left[\mathbf{J}\left(\mathbf{x}^{\prime}, \mathbf{t}^{\prime}\right)\right]_{\mathbf{r e t}}}{r} d \mathbf{x}^{\prime},
$$

with $r=\left|\mathbf{x}-\mathbf{x}^{\prime}\right|$ and $t^{\prime}=t-r / c$. As the current must be evaluated at the retarded time we follow a formalism developed by Lorentz to understand the action of each part of a particle on the others, since we assume it is not 
punctual. The retarded quantity has an expansion in Taylor's series [33]:

$$
[\ldots]_{\text {ret }}=\sum_{n=0}^{\infty} \frac{(-1)^{n}}{n !}\left(\frac{r}{c}\right)^{n} \frac{\partial^{n}}{\partial t^{n}}[\ldots]_{t^{\prime}=t} .
$$

This is a pre-relativistic formulation restraining the validity of its results to low particle velocities. For commodity from now on we decompose the total fields into the external field $\mathbf{A}_{\text {ext }}$ and the self-fields $\mathbf{A}_{s}$ (doing the same as well for $\mathbf{E}$ and $\mathbf{B}$ fields):

$$
\mathbf{A}=\mathbf{A}_{e x t}+\mathbf{A}_{s}
$$

The total linear momentum is conserved only when using the canonical momentum (e.g., Ref. [38]), p, and it is given by

$$
\mathbf{p}=\mu \mathbf{v}+q \mathbf{A}
$$

Our aim is to obtain a consistent approach and hence we use Newton's second law for a charge $q$ in the presence of an external force $\mathbf{F}^{e x t}$. This is a local continuity fluid-like equation of the type:

$$
\frac{d p_{i}}{d t}+I_{i}=0
$$

where in the point-particle limit $I_{i}$ takes the form:

$$
I_{i}=\int\left(d \mathbf{S} \cdot \mathbf{J}_{i}\right)=-\mu \frac{d v_{i}}{d t}-q \frac{D A_{i}}{D t}=-F_{i}^{e x t} .
$$

Here, $\mathbf{J}_{\mathbf{i}}$ is the i-component of a second rank tensor $\mathbb{J}$, and we denote the observable mass by $\bar{m}$ and the mechanical (bare) rest mass by $\mu$. Hence, $\mathbf{J}_{\mathbf{x}}$ is a vector field representing the momentum flux along the $\mathbf{x}$ direction.

Substituting the particle acceleration $\mathbf{a}=d \mathbf{v} / d t$ into Eq. 5], it leads to the dynamical equation:

$$
\mu \mathbf{a}=\mathbf{F}^{e x t}-q \frac{D \mathbf{A}}{D t},
$$

where $D / D t$ means the total (convective) derivative offering a natural frame to describe the motion of an electromagnetic system relatively to an inertial frame. Curiously, Maxwell expressed the electromotive force [39] in the form $\mathbf{E}=-D \mathbf{A} / D t$, although he did not explore fully its consequences which were studied more carefully by others after him [40, 41, 42, 43]. The convective derivative operator in space is given by

$$
\frac{D}{D t}=\frac{\partial}{\partial t}+\mathbf{v} \cdot \frac{\partial}{\partial \mathbf{r}}
$$

After its substitution into Eq. 7], we obtain

$$
\mu \mathbf{a}=\mathbf{F}^{e x t}-q \frac{\partial \mathbf{A}}{\partial t}-q \mathbf{v} \cdot \frac{\partial \mathbf{A}}{\partial \mathbf{r}} .
$$

The magnitude of the force derived by this change - the $q D \mathbf{A} / D t$ term - maybe interpreted as an induced force of inertia acting on the particle. Jefimenko [44] called it the electrokinetic force term (although only considering the partial time derivative). Since $\mathbf{E}_{i}=-\partial \mathbf{A} / \partial t$ when taking due care with of the convective derivative, remark that we can rewrite Eq. 9 also in the form

$$
\mu \mathbf{a}=\mathbf{F}^{e x t}-q \frac{\partial \mathbf{A}}{\partial t}+q[\mathbf{v} \times \mathbf{B}]-q \nabla_{r}(\mathbf{v} \cdot \mathbf{A})
$$

where the B-field appears explicitly. The terms with the self-fields give the reaction force and as well terms of higher order with no clear physical interpretation (e.g., Ref. [33], sec.17.3). The last term in Eq. 10 is related to the Aharonov-Bohm effect [45, 47, 48]. Also, this equation shows us that the particle's own Coulomb field doesn't contribute to a net self-force; when subject alone to its own Coulomb field the extended particle describes a uniform velocity motion. In fact, Eq. 10 contains all the required physical terms contributing to the punctual particle energy and, as it will be shown in the forgoing calculations, the self-force-derived mass equals the electrostatic energy. It is now clear that the source of the " $4 / 3$ problem" resides in previous wrong formulation of the total overall mutual force 
between different elements of charge of the extended charged particle [23, 24], or stating into another way, it is due to the procedure of integration of the self-field of the charge which must refer to field points that move along with the particle [12, 13, 54].

Next, we can apply Eq. 10 to an extended charged particle in its own frame while assuming spherical distribution of charge and slow acceleration. These assumptions probably describe well a charged particle at small velocities. At higher velocities the particle acquires an ellipsoidal shape and our approximation are not anymore valid. To this purpose, our Eq. 9 can be conveniently written under the form:

$$
\mu \mathbf{a}=\mathbf{F}^{e x t}-\int d^{3} x \rho(\mathbf{x}, t) \frac{\partial \mathbf{A}_{\mathbf{s}}(\mathbf{x}, t)}{\partial t}-\int d^{3} x \rho(\mathbf{x}, t)\left(\mathbf{v}(t) \cdot \frac{\partial \mathbf{A}_{s}(\mathbf{x}, t)}{\partial \mathbf{r}}\right) .
$$

Inserting Eq. 2 into Eq. 11 we obtain several terms with interesting physical meaning. The first integral in the right hand side gives the following serial development:

$$
\mathbf{I}_{1}^{n}=-\frac{1}{4 \pi \varepsilon_{0}} \sum_{n=0}^{\infty} \frac{(-1)^{n}}{n ! 2 c^{n+2}} \frac{\partial^{n+1} \mathbf{v}}{\partial t^{n+1}} \int d^{3} x \int d^{3} x^{\prime} \rho(\mathbf{x}, t) r^{n-1} \rho\left(\mathbf{x}^{\prime}, t\right) .
$$

The first two terms of the series are, respectively,

$$
\mathbf{I}_{1}^{0}=-\frac{U_{e s}}{c^{2}} \mathbf{a}
$$

and

$$
\mathbf{I}_{1}^{1}=\frac{e^{2}}{2 c^{3}} \frac{\partial^{2} \mathbf{v}}{\partial t^{2}}=\frac{e^{2}}{2 c^{3}} \dot{\mathbf{a}}
$$

In the above equations we put, $e^{2}=q^{2} /\left(4 \pi \varepsilon_{0}\right)$, and $R$ represents the classical particle radius. A factor $1 / 2$ was inserted into the integrals appearing in Eq. 11 since they represent the interaction of a given element of charge $d q$ with all the other parts, otherwise we count twice that reciprocal action.

In fact, the above derived equations constitute the radiation reaction field. Note that Millonni [49] has shown that from the fluctuation-dissipation theorem there must exist an intimate connection between radiation reaction and the zero-point field (ZPF), since the spectrum of the ZPF depends of the third derivative of the particle's position vector. In this derivation we used the value of the electrostatic energy as given by:

$$
U_{e s}=\frac{1}{2} \int d^{3} x \rho(\mathbf{x}, t) \Phi(\mathbf{x}, t),
$$

where we have used the instantaneous electrostatic potential:

$$
\Phi(\mathbf{x}, t)=\int d^{3} x^{\prime} \frac{\rho\left(\mathbf{x}^{\prime}, t\right)}{4 \pi \varepsilon_{0} r} .
$$

The obtained value is dependent of the assumed structure of the "electron-like" particle with the charge concentrated on the surface of a sphere with radius $R$ [50, 51], while if we assume a charged spherical particle we should obtain instead $U_{e s}=2 e^{2} / 3 c^{2} R$. The Dirac's "bubble-model" of the electron [52] has the advantage to avoid the singularity that otherwise should exists at the center of the sphere and which amount to an infinite energy inside; there is no electric field inside the classical radius.

The application of the Lorentz's procedure to the second integral of Eq. 9 gives:

$$
\mathbf{I}_{2}^{n}=\frac{1}{2} \sum_{n=0}^{\infty} \frac{(-1)^{n}}{n ! c^{n+2}} \int d^{3} x \rho(\mathbf{x}, t) \int d^{3} x^{\prime} \frac{r^{n-2}}{4 \pi \varepsilon_{0}} \frac{\partial^{n} \rho\left(\mathbf{x}^{\prime}, t\right) \mathbf{v}(t)}{\partial t^{n}}\left(\mathbf{v}(t) \cdot \mathbf{u}_{\mathbf{r}}\right)
$$

Here, $\mathbf{u}_{\mathbf{r}}$ denotes the unitary radius vector. The first two terms of the previous power expansion can be readily found:

$$
\mathbf{I}_{2}^{0}=\frac{v^{2}}{c^{2}} \mathbf{F}_{e s}
$$


which gives a null result for a spherical symmetry, but not when the velocity is different from zero (since the symmetry is no longer spherical), and

$$
\mathbf{I}_{2}^{1}=-\frac{1}{2 c^{3}} \int d^{3} x \rho(\mathbf{x}, t) \frac{\partial}{\partial t}\left[v(t) \int d^{3} x^{\prime} \frac{\rho\left(\mathbf{x}^{\prime}, t\right)}{4 \pi \varepsilon_{0} r^{2}}\left(\mathbf{v}(\mathbf{t}) \cdot \mathbf{u}_{\mathbf{r}}\right)\right] .
$$

Note that the $n=3$ term is of order of the second derivative $\sim \partial^{2} \mathbf{v} / \partial t^{2}$, hence negligible when compared to the previous ones under our initial assumptions.

Working along these lines, one is inclined to state that whenever there is a particle with mass $m$ and charge $q$ accelerating or decelerating it will be generated an opposed force given by $\mathbf{F}_{E_{i}}$ which will act against the acceleration vector; indeed, this mechanism derives from the exchange of "potential momentum" between the particle and the field generated by its motion. Due to this interchange between matter and fields, the total particle's mass results to be the sum of the mechanical mass term (which we assume as hypothetically generated by interactions of other nature than electromagnetic) plus the mass of electromagnetic origin (which results from the time-dependent $\mathbf{A}$ vector). The previous development lead us to rewrite Newton's equation of motion for an accelerated "electron-like" extended charge in a first approximation under the form (a dot means a time derivative):

$$
\dot{\mathbf{v}} \bar{m}=\mathbf{F}^{e x t}+\mathbf{F}_{S c h}+\mathbf{F}_{s t} .
$$

Above, the observed rest mass of the extended charged sphere is:

$$
\bar{m}=\mu+\frac{U_{e s}}{c^{2}}=\mu+\frac{e^{2}}{2 R c^{2}} .
$$

Together with some possible external force $\mathbf{F}^{e x t}$, there is the Schott term [53] which is the source of the radiation field, and it is given by:

$$
\mathbf{F}_{S c h}=\frac{e^{2}}{2 c^{3}} \dot{\mathbf{a}} .
$$

It means that the particle experiences a field reaction force when it occurs a change in the acceleration. Finally, the last term represents the stress force which is the source of the deformation of the field lines and it is here given by:

$$
\mathbf{F}_{s t}=-\mathbf{I}_{\mathbf{2}}^{\mathbf{1}} .
$$

It is worth to point out that in Eq. 20 Lorentz covariance is recovered. The analysis made by Hnizdo showed the contributions of the electromagnetic self-field to the energy and momentum of a charge and/or current carrying body and the important role of hidden mechanical momentum [54]. At any rate, it is clear from the previous developments that the electromagnetic mass $m_{e m}$ equals the electrostatic mass $m_{e s}=e^{2} / 2 R c^{2}$ instead of equaling $4 / 3$ the electrostatic mass, as in the Lorentz-Abraham force and power equations [17, 55, 56]. We note that Poincaré pointed out that the stability of the electron required the existence of nonelectromagnetic, attractive forces holding the particle [56] yielding a contribution $m_{0}$ to the total mass, while more recently Puthoff [57] developed a self-consistent, vacuum-fluctuation-based model of the semi-classical electron where an inwardly directed, divergent, electromagnetic vacuum fluctuation radiation pressure compensates the coulomb pressure.

In the framework of classical electrodynamics and under our assumptions, it appears that the electron mass and as well its inertia has possibly an entirely electromagnetic origin, and there is no other kind of contributions to inertia except purely electromagnetic interactions, since one may well put the mechanical mass null, $\mu=0$. This result brings some convenience since whenever we calculate the electron mass with the classical electron radius (which is, however, an adjustable value dependent on the model, see, for example, Ref. [58]), the expression obtained for the electrostatic mass gives exactly the experimental value of the electron mass at rest. As is well known, this viewpoint was defended by Lorentz [9] and Schott [53], being at the same time in agreement with the prior investigations done by Fermi [59] using a variational method which lead him ultimately to show the entire electromagnetic origin of the electron inertial mass. The discrepancies found in literature were shown to be due to faulty electromagnetic momentum- and energy-density expressions [60, 61, 62].

The present formulation embodied in Eq. 11 shows clearly that the inertial force is composed basically of two components: i) the local time derivative of the vector potential and, ii) the convective term on $\mathbf{A}$. The term related to the time derivative of $\mathbf{A}$ in a given point, as we will see, represents a resistance to change induced by the charge acceleration due to the action of its immersed own field (e.g., Ref. [63]). As a matter of fact, the effect of the self-field on the charged particle can be well understood. When an electron suddenly decelerate, the magnetic field increases. According to the induction law, however, an increasing magnetic field gives rise to an electric field. And this same electric field will act on the electron, accelerating it. This effect is interpreted as a contribution to inertia. 
The electrokinetic force is by itself the source of the inertial mass and of the (radiation) field reaction force (or $S c h o t t$ term). The field reaction force contributes to inertia through transfers of energy back and forth between the field and the source (due to the action of the source at the retarded time on itself, see also Ref. [55]). But the electrokinetic force term, which represents a local time derivative of $\mathbf{A}_{\mathbf{s}}$, means that the mass is a locally determined quantity, weakening Mach's conjecture. This result is consistent with the experiments of Hughes and Drever [64] showing that although there is an asymmetrical distribution of matter in the Milky Way galaxy, a directional dependence of inertia mass is negligible $\Delta m / m$. While the electrokinetic term is likely the mass generator it is also reasonable to interpret the term $\mathbf{E}_{\mathbf{i}}$ as the source of the kinetic energy of the particle. But according to Newton's third law, a reaction from "something else" should be present and it is possible that it might come from the physical vacuum, since the experimental findings by Graham and Lahoz [65] implies that the vacuum is the seat of "something in motion", like Maxwell himself envisaged the "aether". Dirac drew attention for the necessity to recuperate the "aether", now renamed physical vacuum, as a necessary tool to understand the interaction of matter with space-time [66].

For illustration of the role of the stress force, let's consider a moving electron along the x-axis. The component of this force term is opposed to the direction of the acceleration acting effectively as a radiation reaction force [67]. The component of this stress force is:

$$
I_{2 x}^{1}=-\frac{e^{2}}{2 R c^{2}} \frac{v_{x}}{c} \frac{\partial v_{x}}{\partial t} .
$$

We get the power consumption by multiplying this (stress) force by the velocity $v$ at time $t=\Delta t$, such as $v=a \Delta t$, and also taking into account that during this interval of time the particle was displaced by $R=v \Delta t$ in this space (medium) where the field lines are build up. The power radiated by an accelerated charge (Larmor's formula) is immediately obtained:

$$
P_{\text {rad }}=-F_{s t} v=\frac{e^{2} a^{2}}{2 c^{3}} .
$$

Our result is consistent with the physical reinterpretation advanced by Harpaz et al [68, 69]. According to these authors the emitted radiation by an accelerated charge is due to the relative acceleration between the electric charge and its own electric field lines that do not move with the charge, but stay in the medium, in contradistinction to the usual (and wrong) argument of the emitted radiation as due to a relative acceleration between the charge and an observer. The result given above for the drag force, however, was obtained in a consistent manner through the continuity equation for canonical momentum applied to an extended charge. In addition, our result is consistent with the mechanism of change in the inertial mass of a system of point charges given by Boyer [45, 46]. Accordingly, it is the electromagnetic fields change during the acceleration of the charges and the concomitant retarded effect which is at the origin of the inertial change of mass, giving at the same time a clear interpretation of the relationship $E=m c^{2}$.

Summarizing our findings, we can state that the convective derivative introduced in the continuity equation for momentum flux (see Eq. 11) traduces not only the conversion from potential to kinetic energy (term $\mathbf{I}_{\mathbf{1}}$ ), but also the convection of potential electromagnetic momentum (term $\mathbf{I}_{\mathbf{2}}$ ), representing the true flux of electromagnetic momentum through the medium, related to the deformation of the vector potential in space and the relativistic increase of mass with velocity. Using an analogy between optics in fluids and the gravitational field [70], we sustain that the charged particle inside the flux of the vector potential acts as submitted to inertial forces (see Eq. 5). We believe that the model exposed in this paper can be helpful to further understand how the physical vacuum interacts with an extended charged particle.

\section{CONCLUSION}

Using the local continuity fluid-like equation for canonical momentum we obtained in a covariant form the dynamical equation of motion of an extended charged particle, subject to the Lorentz's procedure with retarded fields. The total electromagnetic force was introduced taking into account the usual electromagnetic vector and scalar potentials $(\mathbf{A}, \phi)$, since according to quantum mechanics they allow a broader description of physical phenomena, including the Aharonov-Bohm effect and Josephson effect in superconductors.

Our classical electrodynamic approach solve the " $4 / 3$ problem" when taking into due account the total electromagnetic force acting on an extended-charge particle. This is done by introducing the electromagnetic vector and scalar potentials $(\mathbf{A}, \phi)$ and calculating the moving self-field referring to field points that move along with the particle. Using such a procedure the electron mass can be shown to have a complete electromagnetic origin. The local time derivative of $\mathbf{A}$ traduces a conversion from potential to kinetic energy and represents a resistance to motion induced by the rate of change of the acceleration due to the action of its self-field. The velocity dependent term on vector potential 
represents a longitudinal force on the extended charge and as well a stress force acting against the lines of force that is responsible for the relativistic increase of mass.

The explanation of inertia and inertial mass proposed in this paper, in terms of the electromagnetic vector potential and its convective derivative, contributes to a deeper understanding of phenomena related to the classical electromagnetic mass theory.

\section{Acknowledgments}

We would like to thank partial support from the Fundação para a Ciência e a Tecnologia (FCT) and the Rectorate of the Technical University of Lisbon. We would also like to thank important financial support given to one of us (A.A.M) in the form of a PhD Scholarship from FCT.

[1] Max Jammer, Concept of Mass in Classical and Modern Physics (Harvard University Press, Cambridge-Massachustts, 1961)

[2] Leonard Eisenbud, Am. J. Phys. 26144 (1958)

[3] E. Mach, The Science of Mechanics (Open Court, La Salle-Illinois, 1989)

[4] P. W. Bridgman, Am. J. Phys. 2932 (1961)

[5] D. W. Sciama, Mon. Nat. Roy. Astron. Soc. 11334 (1953)

[6] A. D. Sakharov, Sov. Phys. - Doklady, 12(11), 1040 (1968)

[7] H. E. Puthoff, Phys. Rev. A 392333 (1994)

[8] B. Haisch and A. Rueda, Causality and Locality in Modern Physics, Eds. G. Hunter, S. Jeffers, J.-P. Vigier, (Kluwer, Dordrecht, 1998)

[9] Hendrik-Antoon Lorentz, The theory of electrons-and its applications to the phenomena of light and radiant heat (Éditions Jacques Gabay, Paris, 1992)

[10] M. Abraham, Dynamik des Electrons, Nachrichten von der Geselschaft der Wissenschafften zu Göttingen, MathematischPhysikalische Klasse, S. 20

[21] M. Abraham, Phys. Z. 5, 576 (1904)

[12] Amos Ori and Eran Rosenthal, Phys. Rev. D 68, 041701(R) (2003)

[13] Amos Ori and Eran Rosenthal, J. Math. Phys. 45 (6) 2347-2364 (2004)

[14] O. W. Richardson, The Electron Theory of Matter (Cambridge, University Press, 1916)

[15] J. J. Thomson, Philos. Mag. 44294 (1897)

[16] J. J. Thomson, Phil. Mag. 11229 (1881)

[17] Henri Arzeliés, Rayonnement et dynamique du corpuscule chargée fortement accéléré (Gauthier-Villars, Paris, 1966)

[18] G. G. Stokes, Mathematical and Physical Papers (Cambridge WareHouse, Cambridge, UK) Vol. I, pp. 17-19 (1880)

[19] In Einstein's own words: "You know, it would be sufficient to really understand the electron", quoted by Hans G. Dehmlet in his Nobel Lecture, December 8, 1989.

[20] J. D. Prestage, J. J. Bollinger, Wayne M. Itano, and D. J. Wineland, Phys. Rev. Lett. 54 (22) 2387 (1985)

[21] M. Abraham, Phys. Z. 5, 576 (1904)

[22] P. A. M. Dirac, Proc. R. Soc. London, Ser. A 167, 148 (1938)

[23] David J. Griffiths and Russell E. Owen, Am. J. Phys. 51 (12) 1120-1126 (1983)

[24] J. Frenkel, Phys. Rev. E 54 (5), 5859-5862 (1996)

[25] P. Pearle, in Electromagnetism: Paths to Research, ed. D. Teplitz (Plenum, N.Y., 1982), pp. 211-295

[26] Oliver Heaviside, Phil. Trans. Roy. Soc. London, 183A 423 (1893)

[27] Carver A. Mead, Proc. Natl. Acad. Sci. 946013 (1997)

[28] Mark D. Semon and John R. Taylor, Am. J. Phys. 64 (11) 1361 (1996)

[29] Roberto Coisson, Am. J. Phys. 41585 (1972)

[30] E. J. Konopinski, Am. J. Phys. 46(5) 499 (1978)

[31] M. G. Calkin, Am. J. Phys. 47(1) 118 (1979)

[32] Y. Gingras, Am. J. Phys. 48(1) 84 (1980)

[33] J. D. Jackson and L. B. Okun, Rev. Mod Phys. 73663 (2001)

[34] D. Iencinella and G. Matteucci, Eur. J. Phys. 25249 (2004)

[35] Grant R. Fowles, Am. J. Phys. 48(9) 779 (1980)

[36] Akira Tonomura, Proc. Natl. Acad. Sci. 102 (42) 14952 (2005)

[37] Terence W. Barrett 2001 Topological Approaches to Electromagnetism, Modern nonliner Optics (Ed. M. W. Evans), Part3, $2^{\text {nd }}$ Ed., pp 699-734, New York, USA: John Wiley

[38] Landau and Lifschitz, Théorie des Champs, (Editions Mir, Moscow) 1989

[39] J. D. Jackson, L. B. Okun, Rev. Mod. Phys. 73663 (2001)

[40] G. F. C. Searle, Phil. Trans. 187 A 675 (1896) 
[41] Heinrich Hertz, Electric waves - Researches on the propagation of electric action with finite velocity through space (Dover, New York, 1962). English translation of the original German work published in 1892.

[42] Nathan Rosen, David Schieber, Am. J. Phys. 50 (11), 974-976 (1982)

[43] Mario J. Pinheiro, to appear in Phys. Essays 20 (2) [arXix.org physics/0511103]

[44] Oleg D. Jefimenko, Causality Electromagnetic Induction and Gravitation (Electret Scientific Company, Star City, 2000)

[45] Timothy H. Boyer, Phys. Rev. D 8(6) 1679 (1973)

[46] Timothy H. Boyer, Am. J. Phys. 46 (4) 383 (1978); Timothy H. Boyer, Am. J. Phys. 47 (2) 129 (1979)

[47] Y. Aharonov and D. Bohm, Phys. Rev. 115485 (1959)

[48] G. T. Trammel, Phys. Rev. 134(5B) B1183-B1184 (1964)

[49] P. W. Millonni, Am. J. Phys. 52 (4) 340 (1984)

[50] Victor F. Weisskopf, Rev. Mod. Phys. 21 (2) 305 (1949)

[51] F. Rohrlich, Am. J. Phys. 28639 (1960)

[52] P. A. M. Dirac, Proc. Roy. Soc. London Ser. A 26857 (1962)

[53] G. A. Schott, Electromagnetic Radiation (Cambridge U.P., Cambridge, 1912)

[54] V. Hnizdo, Am. J. Phys. 65 (1) 55 (1997)

[55] Arthur D. Yaghjian, Relativistic Dynamics of a Charged Sphere-Updating the Lorentz-Abraham model (Springer-Verlag, Berlin, 1992)

[56] Henri Poincaré, La Mécanique Nouvelle (Editions Jacques Gabay, Paris, 1989)

[57] H. E. Puthoff, Int. J. Theor. Phys. 46 (12), 3005-3008 (2007)

[58] Richard Becker, Electromagnetic Fields and Interactions, Vol. I, p.274 (Dover, New York, 1964)

[59] E. Fermi, Nuovo Cimento 25159 (1923)

[60] C. Möller, Annales de L' Institut Henri Poincaré 11 (5) 251 (1949)

[61] J. W. Butler, Am. J. Phys. 37 (12) 1258 (1969)

[62] W. Wilson, Proc. Phys. Soc. 48736 (1936)

[63] I. Campos and J. L. Jimenez, Am. J. Phys. 57 (7) 610 (1989)

[64] S. Weinberg, Gravitation and Cosmology: Principles and Applications of the General Theory of Relativity (Wiley, New York, 1972), pp. 86-88

[65] G. M. Graham and D. G. Lahoz, Nature, Vol. 285, 154 (1980)

[66] P. A. M. Dirac, Nature 168906 (1951)

[67] F. Rohrlich, Am. J. Phys. 68 (12) 1109 (2000)

[68] Amos Harpaz and Noam Soker, Gen. Rel. Grav. 30 (8) 1217 (1998)

[69] Amos Harpaz and Noam Soker, Found. Phys, 33 (8) 1207 (2003)

[70] U. Leonhardt, P. Piwnicki, Phys. Rev. A 604301 (1999) 